ELECTRONIC RESEARCH ANNOUNCEMENTS OF THE AMERICAN MATHEMATICAL SOCIETY

Volume 12, Pages 53-55 (May 10, 2006)

S $1079-6762(06) 00161-2$

\title{
COBOUNDING ODD CYCLE COLORINGS
}

\author{
DMITRY N. KOZLOV
}

(Communicated by Sergey Fomin)

\begin{abstract}
We prove that the $(n-2)$ nd power of the Stiefel-Whitney class of the space of all $n$-colorings of an odd cycle is 0 by presenting a cochain whose coboundary is the desired power of the class. This gives a very short self-contained combinatorial proof of a conjecture by Babson and the author.
\end{abstract}

\section{Preliminaries}

The study of the following family of complexes has recently been undertaken in connection with equivariant obstructions to graph colorings.

Definition 1.1. For any graphs $T$ and $G$, $\operatorname{Hom}(T, G) \subseteq \prod_{x \in V(T)} \Delta^{V(G)}$ consists of all cells $\sigma=\prod_{x \in V(T)} \sigma_{x}$ such that for any $x, y \in V(T)$, if $(x, y) \in E(T)$, then $\left(\sigma_{x}, \sigma_{y}\right)$ is a complete bipartite subgraph of $G$.

In particular, the cells of $\operatorname{Hom}(T, G)$ are indexed by functions $\sigma: V(T) \rightarrow 2^{V(G)}$ satisfying that additional property, and $\operatorname{dim} \sigma=\sum_{v \in V(T)}(|\sigma(v)|-1)$. We refer the reader to the survey [4 for an introduction to the subject of Hom complexes.

The study of the complexes $X_{r, n}:=\operatorname{Hom}\left(C_{2 r+1}, K_{n}\right), n \geq 3$, has been of special interest. Here for $r \in \mathbb{N}$, we let $C_{2 r+1}$ denote both the cyclic graph with $2 r+1$ vertices and the additive cyclic group with $2 r+1$ elements. The adjacent vertices of $v \in C_{2 r+1}$ get labels $v+1$ and $v-1$. Taking the negative in the cyclic group gives an involution $\gamma$ of the graph with a fixed vertex 0 and a flipped edge $(r, r+1)$. Then $\left(X_{r, n}, \gamma\right)$ is a $\mathbb{Z}_{2}$-space; hence the Stiefel-Whitney characteristic class $w_{1}\left(X_{r, n}\right) \in$ $H^{1}\left(X_{r, n} / \mathbb{Z}_{2} ; \mathbb{Z}_{2}\right)$ of the associated line bundle can be considered.

Theorem 1.2. We have $w_{1}^{n-2}\left(X_{r, n}\right)=0$.

The case $r=1$ was settled by Babson and the author in 2. For $r \geq 2$ and odd $n$, it was proved by the same authors in [3]; see also [4, where the remaining case: $r \geq 2, n \geq 4, n$ is even, was conjectured. The latter was then proved by Schultz in [5, 6]. In the next section we give a short self-contained combinatorial proof of Theorem 1.2 covering all cases: we simply take a cochain representative of $w_{1}^{n-2}\left(X_{r, n}\right)$ and certify that it is a coboundary.

First we fix notation. For $t \in \mathbb{N}$, we set $[t]:=\{1, \ldots, t\}$. For a cell complex $X$, we let $X^{d}$ denote the set of $d$-dimensional cells of $X$. Since we are working over $\mathbb{Z}_{2}$,

Received by the editors March 15, 2006.

2000 Mathematics Subject Classification. Primary 55M35; Secondary 05C15, 57S17.

Research supported by Swiss National Science Foundation Grant PP002-102738/1.

(C)2006 American Mathematical Society 
we may identify $d$-cochains with their support subsets of $X^{d}$. Then, the cochain addition is replaced by the symmetric difference of sets, denoted $\oplus$. For $S \subseteq X_{r, n}^{d}$ the coboundary operator translates to $d S=\bigoplus_{\sigma \in S}\left\{\tau \in X_{r, n}^{d+1} \mid \tau \supset \sigma\right\}$.

If $r$ is even, we set $t:=r / 2$, and $v_{i}:=r-2 i+1$ for $i \in[t]$; otherwise, we set $t:=(r+1) / 2$, and $v_{i}:=r+2 i-1$ for $i \in[t]$. For any $v \in C_{2 r+1}$, we put

$$
\begin{aligned}
& A_{v}:=\left\{\sigma \in X_{r, n}^{n-2} \mid \sigma(v)=[n-1]\right\}, \\
& B_{v}:=\left\{\sigma \in X_{r, n}^{n-3} \mid \sigma(v-1) \cup \sigma(v+1)=[n-1]\right\} .
\end{aligned}
$$

For $S \subseteq X_{r, n}^{d}$, let $q(S):=\bigoplus_{\sigma \in S}\{\{\sigma, \gamma \sigma\}\} \in C^{d}\left(X_{r, n} / \mathbb{Z}_{2}\right)$. We see that $q\left(A_{0}\right)=$ $\emptyset$, and $q(S \oplus T)=q(S) \oplus q(T)$ for any $S, T \subseteq X_{r, n}^{d}$. Furthermore, since $\tau \cap \gamma \tau=\emptyset$ for any $\tau \in X_{r, n}^{d+1}$, we have $q(d S)=d q(S)$ for any $S \subseteq X_{r, n}^{d}$.

It is easy to describe a cochain representing $w_{1}^{n-2}\left(X_{r, n}\right)$. Let $\iota: K_{2} \hookrightarrow C_{2 r+1}$ be given by $\iota(1)=r, \iota(2)=r+1$, where $V\left(K_{2}\right)=[2]$. This induces an algebra homomorphism $\varphi: H^{*}\left(\operatorname{Hom}\left(K_{2}, K_{n}\right) / \mathbb{Z}_{2} ; \mathbb{Z}_{2}\right) \rightarrow H^{*}\left(X_{r, n} / \mathbb{Z}_{2} ; \mathbb{Z}_{2}\right)$. It is well known that $\operatorname{Hom}\left(K_{2}, K_{n}\right) / \mathbb{Z}_{2} \cong \mathbb{R}^{n-2}$. Let $\tau \in \operatorname{Hom}\left(K_{2}, K_{n}\right)^{n-2}$ be given by $\tau(1)=$ $[n-1], \tau(2)=\{n\}$. Since the dual of any cell generates $H^{n-2}\left(\mathbb{R} \mathbb{P}^{n-2} ; \mathbb{Z}_{2}\right)$, we have $w_{1}^{n-2}\left(\operatorname{Hom}\left(K_{2}, K_{n}\right)\right)=[\{\{\tau, \gamma \tau\}\}]$. By functoriality of $w_{1}$ we get $w_{1}^{n-2}\left(X_{r, n}\right)=$ $[\varphi(\{\{\tau, \gamma \tau\}\})]$. Comparing this with our notation we derive $w_{1}^{n-2}\left(X_{r, n}\right)=\left[q\left(A_{r}\right)\right]$.

\section{Proof of Theorem 1.2}

Lemma 2.1. We have $d B_{v}=A_{v-1} \oplus A_{v+1}$ for any $v \in C_{2 r+1}$.

Proof. The cells in $d B_{v}$ are obtained by taking a cell $\sigma \in B_{v}$ and adding $x$ to $\sigma(w)$, for some $x \in[n], w \in C_{2 r+1}$. When $w \neq v \pm 1$, we get a cell $\tau$, which appears in $d B_{v}$ twice: in $d \sigma_{1}$ and in $d \sigma_{2}$, where $\sigma_{1}, \sigma_{2}$ are obtained from $\tau$ by deleting one of the elements from $\tau(w)$. When $w=v \pm 1$, we also get a cell $\tau$, which appears in $d B_{v}$ twice: in $d \sigma_{1}$ and in $d \sigma_{2}$, where $\sigma_{1}, \sigma_{2}$ are obtained from $\tau$ by deleting $\{x\}=\tau(v-1) \cap \tau(v+1)$ either from $\tau(v-1)$ or from $\tau(v+1)$, unless $|\tau(v-1)|=1$ or $|\tau(v+1)|=1$. The latter cells appear once and yield $A_{v-1} \oplus A_{v+1}$.

Proof of Theorem 1.2. Set $K:=\bigoplus_{i=1}^{t} q\left(B_{v_{i}}\right)$; then $d K=\bigoplus_{i=1}^{t} d q\left(B_{v_{i}}\right)=$ $\bigoplus_{i=1}^{t} q\left(d B_{v_{i}}\right)=\bigoplus_{i=1}^{t}\left(q\left(A_{v_{i}-1}\right) \oplus q\left(A_{v_{i}+1}\right)\right)=q\left(A_{r}\right) \oplus q\left(A_{0}\right)=q\left(A_{r}\right)$, hence $w_{1}^{n-2}\left(X_{r, n}\right)=\left[q\left(A_{r}\right)\right]=[d K]=0$.

We remark that Theorem 1.2 implies the Lovász Conjecture: for any graph $G$ and any positive integer $r$, if $\operatorname{Hom}\left(C_{2 r+1}, G\right)$ is $k$-connected, then $\chi(G) \geq k+4$. This conjecture was originally settled by Babson and the author in [1, 3.

Going beyond the mere proof of the Lovász Conjecture, the new ideas and techniques in 1, 2, 3] laid the foundation for all further studies of Hom complexes.

\section{REFERENCES}

1. E. Babson, D.N. Kozlov, Topological obstructions to graph colorings, Electron. Res. Announc. Amer. Math. Soc. 9 (2003), pp. 61-68. MR2029466 (2004i:05044)

2. E. Babson, D.N. Kozlov, Complexes of graph homomorphisms, Israel J. Math. 152 (2006), pp. $285-312$.

3. E. Babson, D.N. Kozlov, Proof of the Lovász Conjecture, Annals of Mathematics (2), in press. arXiv:math.CO/0402395

4. D.N. Kozlov, Chromatic numbers, morphism complexes, and Stiefel-Whitney characteristic classes, in: Geometric Combinatorics, IAS/Park City Mathematics Series 14, in press. arXiv:math. AT/0505563 
5. C. Schultz, A short proof of $w_{1}^{n}\left(\mathrm{Hom}\left(\mathrm{C}_{2 \mathrm{r}+1}, \mathrm{~K}_{\mathrm{n}+2}\right)\right)=0$ for all $n$ and a graph colouring theorem by Babson and Kozlov, 8 pages, 2005. arXiv:math.AT/0507346

6. C. Schultz, The relative strength of topological graph colouring obstructions, 10 pages, 2006.

Institute of Theoretical Computer Science, ETH Zürich, Switzerland

E-mail address: dkozlov@inf.ethz.ch 